\title{
Kulturelle und ästhetische Bildung: Einführung in das Themenheft
}

\author{
Annette Scheunpflug • Manfred Prenzel
}

Kinder malen, singen, erzählen, tanzen und springen; sie betrachten, manipulieren und arrangieren Objekte; sie ahmen einerseits nach und probieren andererseits Neues. Tätigkeiten mit ästhetischen Qualitäten sind selbstverständlicher Teil des kindlichen Alltags, und das lange bevor die Schule oder frühpädagogische Einrichtungen sich der Kinder annehmen. Ästhetische Aktivitäten sind ein selbstverständlicher Aspekt und Teil menschlicher Entwicklung. Sie werden nicht nur in sich verfeinert und erweitert - und lassen somit Lernfortschritte erkennen, sondern wirken zurück auf die Person, ihr Selbstverständnis und ihre Orientierung.

So beeindruckend diese Prozesse ästhetischer Bildung aus einer Außensicht sind, so überraschend ist oft ihr Ende. Denn viele dieser ästhetischen Aktivitäten klingen aus, sie werden von Kindern und Jugendlichen nicht mehr weitergeführt, und dies, obwohl sie viele Gelegenheiten hatten, Anregungen zu erfahren, Vorbilder kennen zu lernen und Rückmeldungen zu erhalten. Andere vertiefen ästhetische Erfahrungen und bringen sie zu einer gewissen Meisterschaft.

Unstrittig ist, dass solche Prozesse der Auswahl, Verengung und Konzentration für fortschreitende Entwicklung und Bildung selbstverständlich sind. Besorgnis wird jedoch dann geäußert, wenn der Eindruck entsteht, in den zu allgemeiner Bildung verpflichteten pädagogischen Einrichtungen würde die Balance von Bildungsgütern und Aneignungsformen zugunsten bestimmter anderer Gegenstandsbereiche verschoben. Wenn außerdem verstärkter Bedarf an Qualifikationen für berufliche Anforderungen oder wirtschaftliche Produktivität angemeldet wird, wächst die Sorge, ästhetische und kulturelle Bildung könnte Stück um Stück marginalisiert werden.

(C) Springer Fachmedien Wiesbaden 2013

Prof. Dr. A. Scheunpflug $(\bowtie)$

Otto-Friedrich Universität Bamberg, Markusplatz 3,

96047 Bamberg, Deutschland

E-Mail: annette.scheunpflug@uni-bamberg.de

Prof. Dr. M. Prenzel

TUM School of Education, TU München, Acrisstraße 21,

80333 München, Deutschland

E-Mail: manfred.prenzel@tum.de 
Diese Sorge wird in Deutschland seit einigen Jahren in der Öffentlichkeit zunehmend artikuliert. Anlass sind Ergebnisse von internationalen Leistungsvergleichen im Schulbereich, die seit fünfzehn Jahren hierzulande große Aufmerksamkeit erfahren, und mit denen die Aufmerksamkeit insbesondere auf Fächer wie Mathematik und Naturwissenschaften gerichtet wird. Die dort beschriebenen Probleme lösten vielfältige und weitreichende Maßnahmen aus, diese Fächer und die zu ihnen erhobenen Ergebnisse stärker zu beachten und zu gewichten, Initiativen zur Unterrichtsentwicklung dort zu fokussieren, fachdidaktische Forschung zu intensivieren, Bildungsstandards zu verankern usw. Weitere Maßnahmen wie die Verkürzung der Schulzeit oder die Einrichtung von Ganztagsschulen, die mit Leistungsvergleichen in Zusammenhang gebracht werden, scheinen die Bedenken zu verstärken, dass die ästhetische und kulturelle Bildung derzeit in Gefahr ist, an Bedeutung zu verlieren.

Die „Sorgendiskussion“ wird zusätzlich durch die Tatsache belastet, dass die Problemlagen in den zum Beispiel bei PISA (Prenzel et al. 2008; Klieme et al. 2010) untersuchten Domänen differenziert und empirisch zuverlässig beschrieben werden können, für Felder der kulturellen und ästhetischen Bildung aber fast keine Befunde vorliegen. Es ist sehr schwer geworden, eine Bildungspolitik, die sich heute auf Evidenz zu stützen sucht, alleine durch Behauptungen und Einschätzungen, nicht aber durch gesicherte Forschungsergebnisse zum Handeln zu veranlassen.

Immerhin: Die Entscheidung, den jüngsten Bildungsbericht (Autorengruppe Bildungsberichterstattung 2012) mit dem Schwerpunktthema „kulturelle Bildung“ einer systematischen Beobachtung zuzuführen, lässt Problembewusstsein bei den Verantwortlichen erkennen. Dennoch zeigt das angesprochene Schwerpunktkapitel mit den vielen offenen Fragen, wie groß der Bedarf an systematischer, empirischer Forschung in den Bereichen der ästhetischen und kulturellen Bildung tatsächlich ist.

Alle, die mit empirischer Forschung vertraut sind, wissen, dass es hier nicht einfach um das Anwenden von empirischen Methoden wie zum Beispiel Fragebogen oder Tests oder um adäquate Stichprobenziehungen geht. Entsprechende technischen Fragen wären relativ schnell in den Griff zu bekommen. Sehr viel komplexer und schwieriger sind zunächst die theoretischen Fragen, die möglicherweise in den vielgestaltigen ästhetischen Domänen (wie Musik oder bildnerisches Gestalten) und ihren Traditionen mit besonderen Schwierigkeiten konfrontiert sind. Um dies an einem Beispiel zu erläutern: Will man Ergebnisse kultureller oder ästhetischer Bildung erfassen, muss man notgedrungen modellieren, was mit „Kompetenz“ oder einem vergleichbaren Konstrukt in dieser Domäne gemeint ist und wie diese strukturiert werden kann. Eine solche Konstruktmodellierung kann mit unterschiedlicher Reichweite und Korngröße betrieben werden. Sie muss aber letztlich durch Kategorien und Merkmalsbeschreibungen getragen werden, die eindeutig, für andere nachvollziehbar sind und sich in Aufgabestellungen, Anforderungen und Lösungswege umsetzen lassen. Nicht zuletzt müssen solche Kompetenzmodelle in den einschlägigen wissenschaftlichen Gemeinschaften zur Diskussion gestellt werden mit dem Ziel, zumindest generelle Übereinstimmungen hinsichtlich der Arbeitsrichtungen zu erhalten.

Das vorliegende Themenheft will zu diesen wichtigen Auseinandersetzungen einen Beitrag leisten. Es versammelt Beiträge von Expertinnen und Experten aus den Bereichen ästhetische und kulturelle Bildung, die dort jeweils einen Bedarf an theoretischer 
und konzeptioneller Weiterentwicklung wahrnehmen. Dies gilt in ähnlicher Weise für die zum Teil sehr unterschiedlichen Arbeitsfelder wie auch für Fragestellungen mit stärker praxis- oder grundlagenorientierten Perspektiven. Ein gemeinsames Anliegen aller Autorinnen und Autoren richtet sich darauf, Fortschritte bei der Erschließung des Feldes der kulturellen und ästhetischen Bildung für empirische Forschung erzielen wollen.

Der Band ist in vier große Teile gegliedert. Im ersten Teil stehen historische und systematische Aspekte kultureller Bildung im Vordergrund. Mit dem Beitrag von Jörg Zirfas werden Aspekte einer Historiografie kultureller Bildung skizziert. Das Thema „Ästhetische Bildung“" wird als Geschichte der Ideen, der Biographien und der Ikonologie entworfen und an einem Beispiel exemplarisch illustriert. Diese Skizze lässt erahnen, was es bedeuten kann, eine Historiographie ästhetischer Bildung zu erarbeiten, in der die Verbindungslinien und Diskontinuitäten zwischen diesen drei Zugängen herausgearbeitet werden. Eckart Liebau umreißt systematische Zugänge zur ästhetischen Bildung. Am Beispiel des Schultheaters entfaltet er die Fruchtbarkeit einer Differenzierung in Produktions-, Werk- und Rezeptionsästhetik und den damit verbundenen Bildungsprozessen. Auch in diesem Beitrag wird auf das Forschungsdesiderat in diesem Feld verwiesen.

Im zweiten Teil des Bandes geht es um empirische Befunde zur Situation kultureller Bildung. Zunächst stellt Anna-Lena Østern die Situation in Norwegen dar. 2010/2011 wurde in Norwegen eine Studie zum Status kultureller Bildung durchgeführt, deren Ergebnisse hier im Überblick dargestellt und kommentiert werden. Zudem werden zahlreiche Forschungs- und Entwicklungsprojekte zur ästhetischen Bildung skizziert. Deutlich wird, welche hohe Bedeutung ästhetische Bildung einnimmt. Deren Konzeptionierung als „,kulturelle Literalität“ führte zu einer starken Beachtung in der didaktischen Debatte sowie in der Lehrerbildung. Einen Blick auf die kulturelle Bildung in der Schweiz erlaubt der Beitrag von Silvia Grossenbacher und Chantal Oggenfuss. Sie stellen die Ergebnisse einer „Trendanalyse“ vor, die sich mit der Situation der Unterrichtsfächer Musik, Kunst und Gestaltung in der Schweiz über eine Dokumentenauswertung sowie Experteninterviews auseinandersetzt. Die Autorinnen berichten über die Ergebnisse einer Lehrplananalyse, die Beziehung der Schule zum Kulturbetrieb sowie die Aus- und Fortbildungen von Lehrerinnen und Lehrern. Zudem wird ein Blick auf die Forschungslandschaft zur kulturellen Bildung der Schweiz geworfen. Vor diesem Hintergrund werden strukturelle Maßnahmen zur Weiterentwicklung der kulturellen Bildung in diesem Land gefordert.

Die nachfolgenden Beiträge thematisieren die Situation kultureller Bildung in Deutschland. Horst Weishaupt und Karin Zimmer erläutern den dem Bildungsbericht zugrundeliegenden Begriff des „Indikators“ und diskutieren die zur Verfügung stehenden Datengrundlagen sowie Konzepte einer datengestützten Darstellung kultureller Bildung. Vor diesem Hintergrund werden Perspektiven für Verbesserungen der Dauerbeobachtung kultureller Bildung in Deutschland umrissen. Einen altersbezogenen Blick auf die Teilhabe an Kultur wagt Susanne Keuchel. In ihrem Beitrag werden die Formen jugendkultureller Teilhabe am kulturellen Leben mit denen der älteren Generationen verglichen. Dabei zeigt sich, dass einerseits Jugendliche sehr offen für neue Kunstrichtungen und kulturelle Angebote sind, andererseits aber in ihrem kulturellen Verhalten abhängig sind von Angebotsstrukturen sowie Vorstellungen kultureller Altersrollen; oft werden beispielsweise klassische Konzerte eher der kulturellen Praxis älterer Menschen zugeordnet. 
An diese beiden Überblicksartikel schließen sich nun Situationsbeschreibungen an, in denen spezifische kulturelle Domänen in den Blick genommen werden. Mariana Grgic und Ivo Züchner berichten aus der Studie „Medien, Kultur und Sport bei jungen Menschen" (MediKuS) über Daten, die Aufschluss über das Spielen von Musikinstrumenten bei Kindern und Jugendlichen geben. Sie können schichtspezifische Unterschiede beim Spielen eines Instruments und der Wahl der Instrumente aufzeigen; hingegen erweisen sich die Orte, an denen Musik gemacht wird, als weniger schichtbezogen. Insbesondere dann, wenn Heranwachsende ein Instrument mit Interesse spielen, weisen die Intensität und Form der Beschäftigung sowie die Motive des Musizierens keine herkunftsspezifischen Unterschiede auf. Diese Studie verweist auf die Bedeutung, die die Schule für die kulturelle Bildung gerade in kulturell distanzierteren Milieus hat, da diese offenkundig eine Angebotsstruktur präsentiert, die - zumindest potenziell - Interesse zu wecken vermag.

Einen anderen Blick auf jugendliche Teilhabe an Kultur erlaubt die qualitative Studie von Ronald Hitzler, Babette Kirchner und Jessica Pahl auf Jugendkulturen in Events. In ihrem Beitrag zeigen sie an zwei Fallbeispielen (einem Festival sowie einem Improvisationstheater) die Verwendung von körperlichen Inszenierungen als soziale Form der Selbstintegration und Unterscheidung. Die Autoren beschreiben das pädagogische Potenzial dieser jugendkulturellen Ausdrucksformen und deren integrative Kraft als „Gesellungsgebilde“ angesichts der Komplexitätssteigerung gesellschaftlicher Wirklichkeitskonstruktionen im Kontext von Pluralisierung und Individualisierung. Im Hinblick auf kulturelle Bildung von Jugendlichen wird häufig unterstellt, dass die Qualität des schulischen Angebots stark durch die Schulart gekennzeichnet sei und Gymnasien deutlich besseres Angebot ermöglichen würden als andere Schularten. Rosa Scherer, Mareike Tarazona und Horst Weishaupt stellen in ihrem Beitrag die diesbezügliche Einschätzung von Schulleitungen dar. Wenn auch die Einschätzungen von Schulleitungen nicht immer einen hinreichenden Eindruck über die schulische Wirklichkeit vermitteln, geben die Ergebnisse dieser Untersuchung zu der Einschätzung Anlass, dass sich mit der Veränderung hin zu Ganztagsschulen die Angebote kultureller Bildung auch außerhalb von Gymnasien deutlich erweitern bzw. diese Schulart im Hinblick auf differentielle kulturelle Bildungsangebote an Einfluss einbüßt. Offensichtlich verliert sich das Engagement für kulturelle Praktiken nicht unmittelbar nach der Schulzeit. Christian Kerst hat künstlerische und ästhetische Aktivitäten Studierender untersucht. Er kann zeigen, dass fast zwei Drittel aller Studierenden einer kulturellen oder künstlerischen Aktivität nachgeht. Allerdings haben die Universitäten daran wenig eigenen Anteil; der größte Teil führt Aktivitäten weiter, die bereits zuvor aufgenommen worden waren, und sie finden auch außerhalb der Hochschule statt.

Kulturelle und ästhetische Bildung ereignet sich auch in Orten der Selbstbildung, wie es beispielsweise Museen sind. Im Beitrag von Annette Noschka-Roos und Doris Lewater wird abschließend ein Überblick über den Diskussionsstand zur Situation kultureller Bildung in Museen gegeben. Hier wird einmal mehr deutlich, wie eng kulturelle Bildung und ästhetisches Lernen miteinander verwoben sind, ist doch das Lernen im Museum untrennbar mit haptischen und sinnlich-ästhetischen Lernerfahrungen in der Präsentation der Objekte verbunden. Die Autoren geben einen Überblick über die Entwicklung der Besucherforschung. Sie zeigen, dass in der Besucherforschung sich mehr und mehr 
die Beobachtung durchsetzt, dass Besuchende sich in Abhängigkeit ihrer kognitiven und motivationalen Merkmale aktiv eine Darstellung erschließen, die einen persönlichen Bedeutungshof entstehen lässt. Vor diesem Hintergrund wird in der Museumsforschung der persönliche Hintergrund für die Lernwirkung von Museumsbesuchen als Orten informellen Lernens betont und der Lernprozess als eine je individuell gestaltete Erfahrung im Umgang mit der Ausstellung und deren Objekten reflektiert.

Im dritten Teil des Bandes steht die häufig mit kultureller Bildung in Verbindung gebrachte These im Mittelpunkt, dass kulturelle Bildung zu Transfereffekten in anderen Bildungsbereichen führt oder sich Auswirkungen auf die Persönlichkeit zeigen.

Der Beitrag von Christian Rittelmeyer zu Transferwirkungen künstlerischer Tätigkeiten befasst sich kritisch mit diesem Anspruch. Er arbeitet heraus, dass eine solche Forschungsfrage einer umfassenden theoretischen Fundierung bedarf. Systematisch wird dieser Zugang an einem Beispiel (malender Kinder) entfaltet und die empirischen Evidenzen daran aufgezeigt, die auf Transfereffekte verweisen. Stefan Kröner entwickelt in seinem Beitrag zur kulturellen Partizipation bei Jugendlichen als Feld der Person-Umwelt-Transaktion ein Rahmenmodell für die Integration bislang kaum verbundener soziologischer, kulturpädagogischer und differentialpsychologischer Forschungsansätze zu kulturellen Aktivitäten. Zentral dafür ist die kulturelle Partizipation als Feld der Transaktion von Person und Umwelt. Er kann das Modell mit vier empirischen Untersuchungen absichern und damit eine brauchbare Operationalisierung für die Transfer-Forschung liefern.

Mit dem vierten Abschnitt dieses Bandes wird ein Blick in die pädagogische Praxis geworfen. Max Fuchs berichtet von der Profilierung von Schulen als Kulturschulen und beschreibt die damit verbundenen Prozesse von Schul- und Unterrichtsentwicklung im Feld der ästhetischen Bildung. Wolfgang Zacharias beschreibt Herausforderungen und Möglichkeiten kulturell-ästhetischer Medienbildung unter den Bedingungen von web2.0 und entwickelt dafür theoretische wie konzeptionelle Perspektiven.

Der vorliegende Band geht zurück auf das Forum 2011 zur ästhetischen Bildung, das die Redaktion der ZfE gemeinsam mit dem herausgebenden Verlag organisierte. Wir danken den Teilnehmenden des Forums für die engagierte Diskussion, die in die vorliegenden Beiträge eingeflossen sind. Dem VS-Verlag und der FU-Berlin gebührt herzlicher Dank für die Unterstützung der Tagung. Sabine Lang sei sehr herzlich für die redaktionelle Arbeit gedankt.

\section{Literatur}

Autorengruppe Bildungsberichterstattung. (2012). Bildung in Deutschland 2012. Ein indikatorengestützter Bericht mit einer Analyse zur kulturellen Bildung im Lebenslauf. Bielefeld: Bertelsmann Verlag.

Klieme, E., Artelt, C., Hartig, J., Jude, N., Köller, O., Prenzel, M., Schneider, W., \& Stanat, P. (Hrsg.). (2010). PISA 2009. Bilanz nach einem Jahrzehnt. Münster: Waxmann.

Prenzel, M., Artelt, C., Baumert, J., Blum, W., Hammann, M., Klieme, E., \& Pekrun, R. (Hrsg.). (2008). PISA 2006 in Deutschland. Die Kompetenzen der Jugendlichen im dritten Ländervergleich. Münster: Waxmann. 